

\title{
Transferability of neural network potentials for varying stoichiometry: Phonons and thermal conductivity of Mn $\mathrm{x}$ Ge y compounds
}

Claudia Mangold, Shunda Chen, Giuseppe Barbalinardo, Jörg Behler, Pascal Pochet, Konstantinos Termentzidis, Yang Han, Laurent Chaput, David Lacroix, Davide Donadio

\section{To cite this version:}

Claudia Mangold, Shunda Chen, Giuseppe Barbalinardo, Jörg Behler, Pascal Pochet, et al.. Transferability of neural network potentials for varying stoichiometry: Phonons and thermal conductivity of Mn x Ge y compounds. Journal of Applied Physics, 2020, 127, 10.1063/5.0009550 . hal-02878286

\author{
HAL Id: hal-02878286 \\ https://hal.science/hal-02878286
}

Submitted on 23 Jun 2020

HAL is a multi-disciplinary open access archive for the deposit and dissemination of scientific research documents, whether they are published or not. The documents may come from teaching and research institutions in France or abroad, or from public or private research centers.
L'archive ouverte pluridisciplinaire $\mathbf{H A L}$, est destinée au dépôt et à la diffusion de documents scientifiques de niveau recherche, publiés ou non, émanant des établissements d'enseignement et de recherche français ou étrangers, des laboratoires publics ou privés. 


\section{Transferability of neural network potentials for varying stoichiometry: Phonons and thermal conductivity of $\mathrm{Mn}_{x} \mathrm{Ge}_{y}$ compounds}

F

Cite as: J. Appl. Phys. 127, 244901 (2020); https://doi.org/10.1063/5.0009550

Submitted: 31 March 2020 . Accepted: 22 May 2020 . Published Online: 22 June 2020

Claudia Mangold, Shunda Chen (D), Giuseppe Barbalinardo (D), Jörg Behler (D), Pascal Pochet (D), Konstantinos Termentzidis (D), Yang Han (D), Laurent Chaput, David Lacroix (iD), and Davide Donadio (iD)

\section{COLLECTIONS}

Note: This paper is part of the special collection on Machine Learning for Materials Design and Discovery

F This paper was selected as Featured
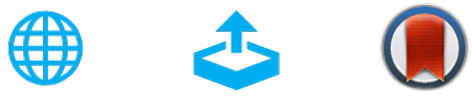

\section{Lock-in Amplifiers up to $600 \mathrm{MHz}$}
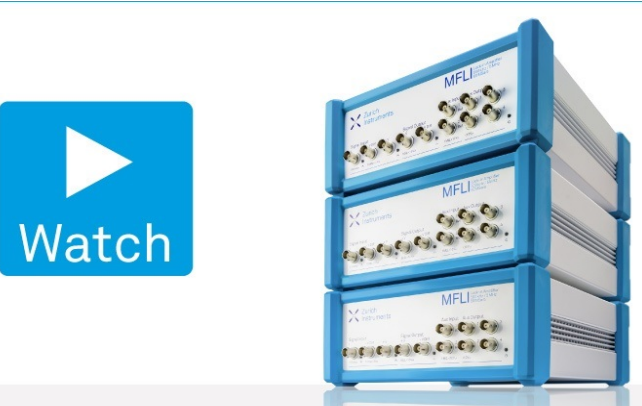


\title{
Transferability of neural network potentials for varying stoichiometry: Phonons and thermal conductivity of $\mathrm{Mn}_{x} \mathrm{Ge}_{y}$ compounds
}

Cite as: J. Appl. Phys. 127, 244901 (2020); doi: 10.1063/5.0009550

Submitted: 31 March 2020 - Accepted: 22 May 2020 .

Published Online: 22 June 2020

Claudia Mangold, ${ }^{7}$ Shunda Chen, ${ }^{2}$ (D) Giuseppe Barbalinardo, ${ }^{2}$ (D) Jörg Behler, ${ }^{3}$ (iD Pascal Pochet, ${ }^{4}$ (D) Konstantinos Termentzidis, ${ }^{5}$ (D) Yang Han, ${ }^{6}$ (D) Laurent Chaput, ${ }^{6}$ David Lacroix, ${ }^{6, a)}$ (iD) and Davide Donadio ${ }^{2, b)}$ (iD

\author{
AFFILIATIONS \\ ${ }^{7}$ Max-Planck-Institut für Polymerforschung, Ackermannweg 10, 55128 Mainz, Germany \\ ${ }^{2}$ Department of Chemistry, University of California Davis, One Shields Ave., Davis, 95616 California, USA \\ ${ }^{3}$ Universität Göttingen, Institut für Physikalische Chemie, Theoretische Chemie, Tammannstr. 6, 37077 Göttingen, Germany \\ ${ }^{4}$ Department of Physics, IRIG, Univ. Grenoble Alpes and CEA, F-38000 Grenoble, France \\ ${ }^{5}$ Université Claude Bernard Lyon 1, CNRS, INSA-Lyon, CETHIL UMR5008, F-69621, Villeurbanne, France \\ 'Université de Lorraine, CNRS, LEMTA, Nancy F-54500, France
}

Note: This paper is part of the special collection on Machine Learning for Materials Design and Discovery

a)david.lacroix@univ-lorraine.fr

${ }^{b)}$ Author to whom correspondence should be addressed: ddonadio@ucdavis.edu

\begin{abstract}
Germanium manganese compounds exhibit a variety of stable and metastable phases with different stoichiometries. These materials entail interesting electronic, magnetic, and thermal properties both in their bulk form and as heterostructures. Here, we develop and validate a transferable machine learning potential, based on the high-dimensional neural network formalism, to enable the study of $\mathrm{Mn}_{x} \mathrm{Ge}_{y} \mathrm{materials}$ over a wide range of compositions. We show that a neural network potential fitted on a minimal training set reproduces successfully the structural and vibrational properties and the thermal conductivity of systems with different local chemical environments, and it can be used to predict phononic effects in nanoscale heterostructures.
\end{abstract}

Published under license by AIP Publishing. https://doi.org/10.1063/5.0009550

\section{INTRODUCTION}

Machine learning potentials (MLPs) provide a versatile tool to study complex materials with diverse local chemical environment with accuracy comparable to that of the electronic structure calculations to which they are trained, which is usually density functional theory (DFT). ${ }^{1}$ A few classes of MLPs can actually achieve such level of accuracy and transferability across various states of matter: successful examples are neural network potentials (NNPs), ${ }^{2-4}$ Gaussian approximation potentials (GAPs) with smooth overlap of atomic positions (SOAPs), ${ }^{5,6}$ moment tensor potentials, ${ }^{7}$ and spectral neighbor analysis potentials. ${ }^{8}$ The flexible form and extensive number of parameters of these potentials enable accurate simulations of elemental and binary materials across their phase diagram, including high-pressure phases, ${ }^{9}$ liquids ${ }^{10,11}$ and glasses, ${ }^{12,13}$ interfaces, ${ }^{14}$ and nanostructures. ${ }^{15,16}$ Besides structural stability and total energy, MLPs allow one to model response functions, such as vibrational spectra, ${ }^{17}$ and transport coefficients, e.g., thermal conductivity. ${ }^{18-20}$

Whereas several works proved the efficacy of MLPs in dealing with diverse chemical environments, so far their performance for large variations of stoichiometry in solids has not been systematically tested. Here, we address the transferability of a NN potential of a binary system over a wide range of compositions. For this purpose, we consider $\mathrm{Mn}_{x} \mathrm{Ge}_{y}$, which is an interesting material with several stable and metastable polymorphs, and potential applications in electronics, spintronics, and thermoelectric energy conversion. $^{21-25}$ In particular, among the crystalline phases, MnGe is a fascinating topological material, for which it was recently 
measured a large magneto-thermopower, ${ }^{26}$ but its thermal conductivity is unknown. Ultimately, it would be desirable to attain a reliable description of nanostructured $\mathrm{Mn}$-doped germanium materials. Experiments suggested that $\mathrm{Mn}_{5} \mathrm{Ge}_{3}, \mathrm{Mn}_{11} \mathrm{Ge}_{8}$, and $\mathrm{MnGe}$ play an important role in the formation of $\mathrm{Mn}-\mathrm{Ge}$ phases precipitated in Ge. $\mathrm{Mn}_{5} \mathrm{Ge}_{3}$ and $\mathrm{Mn}_{11} \mathrm{Ge}_{8}$ are both stable under standard pressure and temperature conditions and they exhibit structural similarities. ${ }^{22,24}$

As we focus on the vibrational properties and heat transport of these systems, our goal is to fit and test a NNP that reproduces correctly the structure, the phonon dispersion relations, and the thermal conductivity of the phases of $\mathrm{Mn}_{x} \mathrm{Ge}_{y}$ from pure Ge all the way to $\mathrm{Mn}_{5} \mathrm{Ge}_{3}$, including the magnetic materials $\mathrm{MnGe}$ and $\mathrm{Mn}_{11} \mathrm{Ge}_{8}$, so as to enable future studies of growth, structural transformations and heat transport in nanostructured Mn-doped Ge films. The training set for the NNP is obtained by accurate DFT calculations of total energies and forces. We validate the accuracy and transferability of the NNP by comparing the structural parameters, e.g., equilibrium density and lattice parameters, and the elastic response to hydrostatic compression against those obtained from the calculations of the equations of states of the different $\mathrm{Mn}_{x} \mathrm{Ge}_{y}$ systems by DFT. Phonon dispersion relations are validated against those computed by DFT and the lattice thermal conductivity is compared to that obtained by first-principles anharmonic lattice dynamics and the Boltzmann transport equation. ${ }^{27} \mathrm{We}$ prove that NNPs enable the calculation of thermal conductivity at room temperature by equilibrium molecular dynamics (EMD) and the Green-Kubo approach. ${ }^{28,29}$

\section{SYSTEMS AND METHODS}

\section{A. $\mathrm{Mn}_{x} \mathrm{Ge}_{y}$ compounds}

The properties of the stable phases of $\mathrm{Mn}_{x} \mathrm{Ge}_{y}$ compounds as a function of their composition are thoroughly described by Arras et al. ${ }^{22,24}$ The phase diagram is comprised of 16 known phases with composition between pure Ge and pure Mn. Six of them are stable at ambient conditions, while the others are either stabilized by either temperature or pressure and are metastable at ambient temperature and pressure. In the interval of stoichiometric composition of interest to our research, i.e., from $\mathrm{Ge}$ to $\mathrm{Mn}_{5} \mathrm{Ge}_{3}$, only $\mathrm{Mn}_{11} \mathrm{Ge}_{8}$ is stable at ambient conditions, while $\mathrm{MnGe}_{4}$, $\mathrm{MnGe}$, and $\mathrm{Mn}_{3} \mathrm{Ge}_{5}$ are stable at high pressures. In this work, we consider the three stable phases, bulk $\mathrm{Ge}, \mathrm{Mn}_{11} \mathrm{Ge}_{8}$, and $\mathrm{Mn}_{5} \mathrm{Ge}_{3}(\eta)$, and metastable $\mathrm{MnGe}$ (Fig. 1). Ge, MnGe, and (a)



(c)

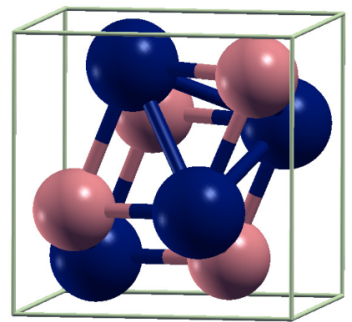

(b)

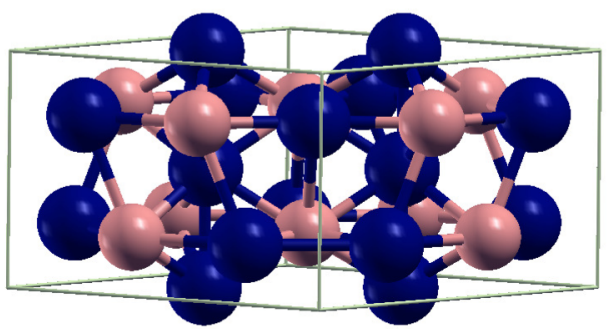

(d)



FIG. 1. Bulk structures: $\mathrm{Ge}(\mathrm{a}), \mathrm{Mn}_{5} \mathrm{Ge}_{3}$ (b), $M n G e(c)$, and $\mathrm{Mn}_{11} \mathrm{Ge}_{8}$ (d). 
$\mathrm{Mn}_{5} \mathrm{Ge}_{3}(\eta)$ have small unit cells consisting of 2, 8, and 16 atoms, while $\mathrm{Mn}_{11} \mathrm{Ge}_{8}$ forms a lower-symmetry structure with 76 atoms per cell. We then decided to focus on the first three structures to train and validate the NNP and eventually to test the transferability to $\mathrm{Mn}_{11} \mathrm{Ge}_{8}$ which was excluded from the training set. Since the NNP used here is constructed as a sum of environmentdependent atomic energies, we expect it to successfully describe also this latter system, because the local chemical bonding environment is quite similar to that of $\mathrm{Mn}_{5} \mathrm{Ge}_{3}(\eta)$.

Except for bulk Ge, the $\mathrm{Mn}_{x} \mathrm{Ge}_{y}$ phases considered exhibit magnetic ordering at low temperature. $\mathrm{Mn}_{5} \mathrm{Ge}_{3}$ is ferromagnetic (FM), as consistently shown both by experiments and DFT calculations. ${ }^{24,30,31}$ The magnetic ordering of MnGe is debated, as early experiments singled it out as antiferromagnetic, while more recent calculations, including the present work, suggest that ferromagnetic ordering is slightly more stable, by about $0.4 \mathrm{eV}$ per unit cell. ${ }^{24}$ More recent neutron scattering experiments suggest a chiral magnetic ordering that is beyond the scope of this work to explore further. ${ }^{32} \mathrm{Mn}_{11} \mathrm{Ge}_{8}$ is an anti-ferromagnet at low temperature, which then converts into a ferromagnet above the Néel temperature of $150 \mathrm{~K}$ and eventually becomes paramagnetic above the Curie temperature of $274 \mathrm{~K}^{33}$ Our unrestricted spin-polarized calculations confirm the antiferromagnetic ordering of $\mathrm{Mn}_{11} \mathrm{Ge}_{8}$ at zero temperature.

\section{B. First-principles calculations}

We use DFT both to fit and to validate our NN potential. DFT calculations, including structural relaxations of unit cells as well as $\mathrm{MD}$ runs and single point calculations of structures along MD trajectories, are performed within the generalized gradient approximation (GGA) by Perdew, Burke, and Ernzerhof (PBE), ${ }^{34}$ as implemented in the plane wave code Quantum-Espresso. ${ }^{35}$ The plane waves basis set is cut off at $55 \mathrm{Ry}$ and the integration on the first Brillouin zone is carried out on uniform Monkhorst-Pack meshes of $k$-points ${ }^{36}$ chosen so as to ensure that the total energy is converged within $0.005 \mathrm{eV} /$ atom for each system. The electronic occupation is smeared according to the Marzari-Vanderbilt scheme with a broadening of $0.05 \mathrm{eV}$, so as to achieve more efficient convergence of metallic systems. ${ }^{37}$ The core electrons are described with the projector augmented wave (PAW) method. ${ }^{38}$ The PAW pseudopotential for Mn was generated using the ATOMPAW code. ${ }^{39}$ We applied an augmentation radius of $1 \AA$ to define the region where the ultrasoft pseudopotential is effective. Mn pseudopotentials are set to treat semi-core electrons explicitly, which is essential to provide the correct level degeneracy for the $\mathrm{Mn}$ atom in vacuum. ${ }^{24}$ Some $\mathrm{Mn}_{x} \mathrm{Ge}_{y}$ compounds exhibit magnetic behavior, as they are either ferromagnetic, anti-ferromagnetic, or non-collinear (chiral); thus, to account for magnetic ordering, we perform unrestricted spin-polarized calculations.

$A b$ initio phonon dispersion relations are calculated using either density functional perturbation theory (DFPT) ${ }^{40}$ or the frozen phonon approach, in which the force constants are computed by finite differences over atoms displacements in a sufficiently large supercell. Given the short-range nature of the forces in the systems considered, both methods provide results with comparable accuracy, as we verified for bulk Ge. While DFPT is more general, it becomes more computationally expensive for crystals with large number of atoms in the unit cell. Hence, for $\mathrm{Mn}_{5} \mathrm{Ge}_{3}$ and $\mathrm{Mn}_{11} \mathrm{Ge}_{8}$, we employ the frozen phonons approach with displacements of the atoms of $0.01 \AA$.

Thermal conductivity is calculated using phono $3 p y,{ }^{27}$ which implements the solution to the linearized Boltzmann transport equations (BTE) in the relaxation time approximation. Second and third order interatomic force constants (IFCs) are computed by DFT using the PAW method as implemented in the VASP code. ${ }^{41-46}$ To obtain forces, total energies are converged with an accuracy better than $10^{-8} \mathrm{eV}$ in supercells whose size is given in Table I.

The BTE is then solved in the relaxation time approximation, which allows one to express the lattice thermal conductivity as

$$
\kappa=\frac{1}{V N_{q}} \sum_{i, q} c_{i}(q) \mathrm{v}_{i}(q) \otimes \mathrm{v}_{i}(q) \tau_{i}(q) .
$$

$\mathrm{v}_{i}(q)$ are the phonon group velocities obtained as $\frac{\partial \omega_{i}(q)}{\partial q}$ and $c_{i}(q)$ is the phonon heat capacity, i.e., $c_{i}(q)=\hbar \omega_{i}(q) \frac{\partial n(\omega, T)}{\partial T}$ with $n$ being the Bose-Einstein distribution function. $\omega_{i}(q)$ and $\mathrm{v}_{i}(q)$ can be obtained from the harmonic force constants alone, but to compute $\tau_{i}(q)$, the third order anharmonic force constants are required. ${ }^{2}$

\section{Neural network potential: Details and training}

We employ the Behler-Parrinello NNP scheme $e^{2,47,48}$ to generate a transferable linear-scaling MLP for germanium and Ge-Mn systems. This general neural network scheme consists of a set of symmetry functions ${ }^{49}$ that feed the atomic coordinates to a number of hidden layers, which provide an analytical expression of the energy. Forces are obtained as the analytical negative gradient of the energy function. For our systems, we chose a relatively simple

TABLE I. Database of structures used to train and test the neural network potential. While we generated an extensive database of structures, we tried to find a minimal training set, so as to avoid over-fitting problems and reduce the number of correlated structures.

\begin{tabular}{lccr}
\hline \hline Species & Cell & Supercell (atoms) & Training set \\
\hline $\mathrm{Ge}$ & fcc & $2 \times 2 \times 2(64)$ & 2522 \\
$\mathrm{Mn}_{5} \mathrm{Ge}_{3}$ & hex. & $1 \times 1 \times 2(32)$ & 2396 \\
$\mathrm{MnGe}$ & sc & $2 \times 2 \times 2(64)$ & 1340 \\
$\mathrm{Ge} / \mathrm{Mn}_{5} \mathrm{Ge}_{3}$ & hex. & $1 \times 1 \times 1(44)$ & 731 \\
& & Total & 4794 \\
\hline \hline
\end{tabular}


architecture using the code RuNNer. ${ }^{48,50}$ It consists of two hidden layers, each containing 20 nodes. For each node, a hyperbolic tangent is used as non-linear "activation function," while the identity $f(x)=x$ is used as activation function for the output layer. 48 atom-centered symmetry functions represent chemical environment of each atom up to a cutoff $r_{c}=6.35 \AA$. A cutoff function is defined so that the potential goes to zero with continuous first and second derivatives at $r_{c}{ }^{49}$ The NNP constructed in this way is short-range, hence the linear scaling. As it neglects long-range, it may not be suitable to treat strongly ionic systems, but this is not the case for $\mathrm{Mn}-\mathrm{Ge}$. A similar approach was employed to fit a NNP for GeTe, ${ }^{51}$ showing excellent performance in describing the structure of its liquid and amorphous phases and the crystallization mechanism, ${ }^{15,52}$ as well as the structure and vibrational properties of nanowires. ${ }^{53}$ Whereas in principle, it would be possible to use larger $\mathrm{NN}$, with more layers or more nodes per layer, that would mean adding even more parameters to fit, thus making it very difficult to achieve accuracy with a limited training set.

Fitting a reliable NNP requires a comprehensive training database. As we are addressing a range of stoichiometry from pure Ge to $\mathrm{Mn}_{5} \mathrm{Ge}_{3}$, besides these two compositions, we include in the database also crystalline $\mathrm{MnGe}$ as a system with intermediate stoichiometry.
Furthermore, to extend the applicability to nanostructured materials, e.g., superlattices $\mathrm{Ge} / \mathrm{Mn}_{x} \mathrm{Ge}_{y}$ and $\mathrm{Mn}_{5} \mathrm{Ge}_{3}$ nanoinclusions in bulk Ge, we add to the training set a superlattice that features $\mathrm{Ge}[111] / \mathrm{Mn}_{5} \mathrm{Ge}_{3}[001]$ interfaces. This superlattice consists of a thin Ge [111] slab $(\sim 7 \AA)$ and an even thinner $\mathrm{Mn}_{5} \mathrm{Ge}_{3}$ slab oriented in the [001] direction. These two surfaces form a clean interface with good lattice matching $(\sim 6 \%)$, which was observed in epitaxial growth experiments. ${ }^{54-56}$ The resulting interfacial structures contain 44 atoms in its unit cell [structure (a) in Fig. 2, while the larger models represented in panels (b) and (c) are used for calculations only]. These structures feature low-energy and low-strain interfaces between $\mathrm{Ge}$ and $\mathrm{Mn}_{5} \mathrm{Ge}_{3}$ : the features of these interfaces favor phase separation of $\mathrm{Mn}-\mathrm{Ge}$ solid solutions leading to the formation of nanostructured films. ${ }^{30}$

To generate a sufficient number of training structures, we performed Born-Oppenheimer Molecular Dynamics (BOMD) simulations of supercells containing about 100 atoms obtained replicating the crystalline structures. BOMD simulations in the constant volume canonical (NVT) ensemble are performed for systems at various densities and temperatures between 300 and $700 \mathrm{~K}$. We stress that it is important to use systems at different densities to train the NNP on larger variations of bond lengths and angles. In particular, we (a)

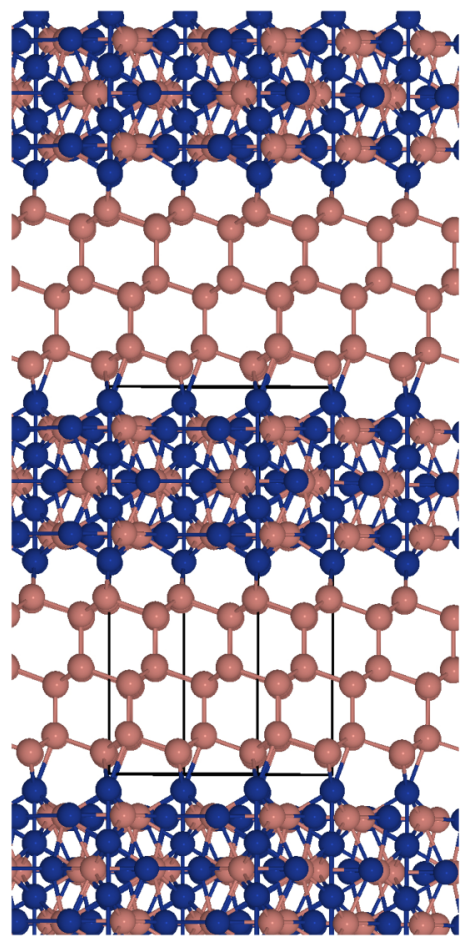

(b)

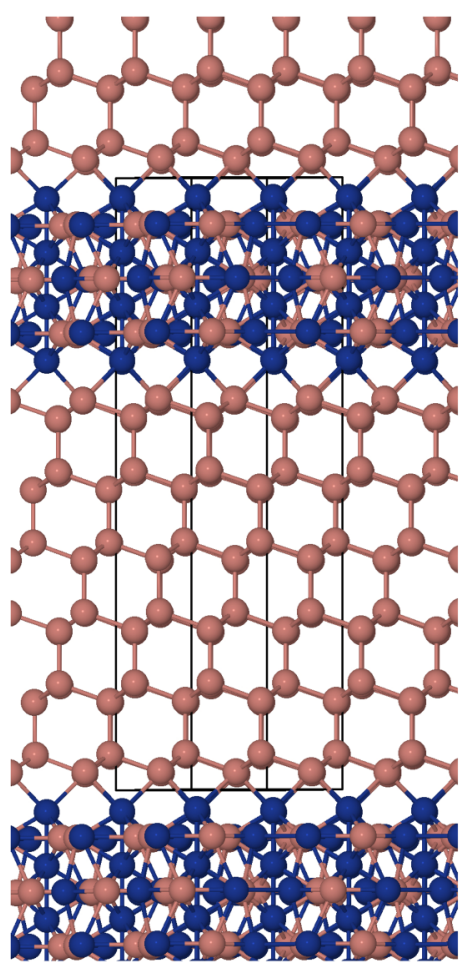

(c)



FIG. 2. Ge[111] $/ \mathrm{Mn}_{5} \mathrm{Ge}_{3}[001]$ heterostructures with varying thicknesses of the Ge layer, namely, $7 \AA$ (a), $18 \AA$ (b), and $28 \AA$ (c) (black boxes correspond to the respective unit cells). 
perform MD simulations for systems with lattice parameters up to $\pm 4 \%$ of the equilibrium lattice constants. In these simulations, the electronic structure is computed at the $\Gamma$-point only, and Newton's equations of motion are integrated with a time step of $4 \mathrm{fs}$. This setup is sufficient to sample the configurational space of the system. From the BOMD trajectories, we extract statistically uncorrelated frames-approximately one every $100 \mathrm{fs}$ of MD trajectory, with a randomized time lag-, for which we perform well-converged electronic structure calculations, as described in Sec. II B. These calculations provide accurate total energies and forces that are both used to train the NNP. Since we consider very different models, with different numbers of atoms of the two types, total energies would not be comparable across the training set. For this reason, we fit the NNP on atomization energies, which have a well-defined physical meaning and are consistent for different systems. Further training configurations are produced while testing the NNP in MD runs, when configurations occur that are out of the interpolation range of the NNP, so as to add cyclical self-consistence to the training procedure.

Since for each frame the number of force components, corresponding to the number of degrees of freedom of the system, overwhelms the single total energy entry, only a randomly chosen fraction of the force components is used to fit the NNP. The best fit is obtained a fraction of the forces corresponding to approximately 3 times the number of total energies. The parameter optimization is carried out using an adaptive Kalman filter. ${ }^{57}$ With this optimization algorithm, the NN parameters are updated upon the presentation of each individual energy or force component, so that a global loss function, which would combine errors from the energies and the forces, is not required. To obtain a balanced statistical weight of the input data, we define an error threshold on energies and forces, and we update the NN parameters only when the error exceeds the threshold. In this way, the optimization process selects and gives more weight to the configurations that are less accurately represented. The error threshold itself is not fixed but specified with respect to the current root mean square error (RMSE) such that the threshold decreases along with the improvement of the fit. To avoid overfitting, we implemented an iterative search of structures that are not well represented, that is, those for which different NNs trained to the same dataset predict very different energies and forces. This search is carried out iteratively while improving the potential until convergence is reached. In addition, we continuously apply the early stopping method, i.e., not all available training points are used for optimizing the NN parameters, but a part of the dataset is kept as an independent test or validation set to assess the quality of predictions for new structures. ${ }^{50}$

Details of the training set used to generate the NNP are given in Table I. This setup leads to a root mean square error (RMSE) of $2.2 \mathrm{meV} /$ atom on the energies of the training set and $2.5 \mathrm{meV} /$ atom for the test set, and it gives a RMSE of $0.085 \mathrm{eV} / \AA$ and $0.089 \mathrm{eV} / \AA$ for the forces on the training and test sets, respectively. Energies per atom obtained with the NNP against the DFT reference are shown in Fig. 3(a) for both the training and the test set. Figure 3(b) shows that the error on the forces is nearly equivalent for all systems: although slightly larger deviations from the DFT reference occur for $\mathrm{Mn}_{5} \mathrm{Ge}_{3}$, the RMSE on the forces is actually similar for the four structures considered. The parameters of the NNP are available as data files in the supplementary material. These are plain ASCII files, in a format readable to the code RuNNer, and consist of a commented input file for RuNNer, the weights of the nodes of the NN layers, and the scaling factors for normalizing the range of the symmetry functions.

Former works adopted very large databases of structures for the NNP Training, ${ }^{51,58-60}$ for example, the NNP for GeTe, ${ }^{51}$ which has the same symmetry functions and similar network structure as ours, was fitted for more than 30000 structures. Here, however, we try to find a minimal database with about one-tenth of the structures. Whereas on one side, we have a range of compositions, on the other side, we can focus for the moment on crystalline structures and superlattices, thus limiting the need for transferability to an extremely broad range of chemical environments. Therefore, we started the refinement process with MD simulations with a preliminary NN potential based on only several hundreds of structures per $\mathrm{Mn}-\mathrm{Ge}$ phase. Problematic structures from these MD runs were picked and added to the training set in order to systematically improve the NNP.

\section{Thermal conductivity from molecular dynamics simulations}

The fitted NNP is exploited to perform MD simulations and to compute the lattice thermal conductivity from the fluctuation of the heat current at equilibrium using the Green-Kubo expression for transport coefficients,

$$
\kappa_{\alpha \beta}=\frac{V}{k_{B} T^{2}} \int_{0}^{\infty}\left\langle J_{\alpha}(0) J_{\beta}(t)\right\rangle d t
$$

where $\kappa_{\alpha \beta}(\alpha \beta=x, y, z)$ is the $\alpha \beta$ th component of the thermal conductivity tensor, $V$ is the volume of the simulation cell, $k_{B}$ is the Boltzmann constant, $T$ is temperature, and $J_{\alpha}$ is the $\alpha$ th component of the heat current vector.

The heat current consists of the sum of a kinetic and a potential energy term,

$$
\mathbf{J}=\mathbf{J}_{k i n}+\mathbf{J}_{p o t}=\sum_{i} E_{i} \mathbf{v}_{i}+\sum_{i} \mathbf{r}_{i} \frac{d E_{i}}{d t} .
$$

The NNP is a short-range analytical function of the coordinates of the system, and the use of atom centered symmetry functions is chosen so that the total energy of the system is expressed as the sum of atomic contributions: $E_{t o t}=\sum_{i} E_{i}$. This observation is sufficient for us to deal with the kinetic term, which usually provides a negligible contribution to the total thermal conductivity of solids. Furthermore, the symmetry functions amount to pair $\left[f\left(r_{i j}\right)\right]$ and three-body functions $\left[f\left(r_{i j}, r_{i k}, r_{j k}\right)\right]$, where $r_{i j}$ indicates the vector connecting two atoms within the chosen cutoff $r_{c}$. ${ }^{1}$ These features of the NNP allow us to define a pairwise force $\mathbf{F}_{i j}$ between two atoms $i$ and $j$, which includes all the three-body contributions. This pairwise force is defined so as to satisfy two conditions: the total force on atom $i$ is given by $\mathbf{F}_{i}=\sum_{j \neq i} \mathbf{F}_{i j}$ and Newton's third law holds, i.e., $\mathbf{F}_{i j}=-\mathbf{F}_{j i}$. These conditions allow us to define a pairwise atomic stress tensor $\sigma_{i}=-\frac{1}{2} \sum_{j \neq i} \mathbf{r}_{i j} \otimes \mathbf{F}_{i j}$, which, in turn, can be 
(a)

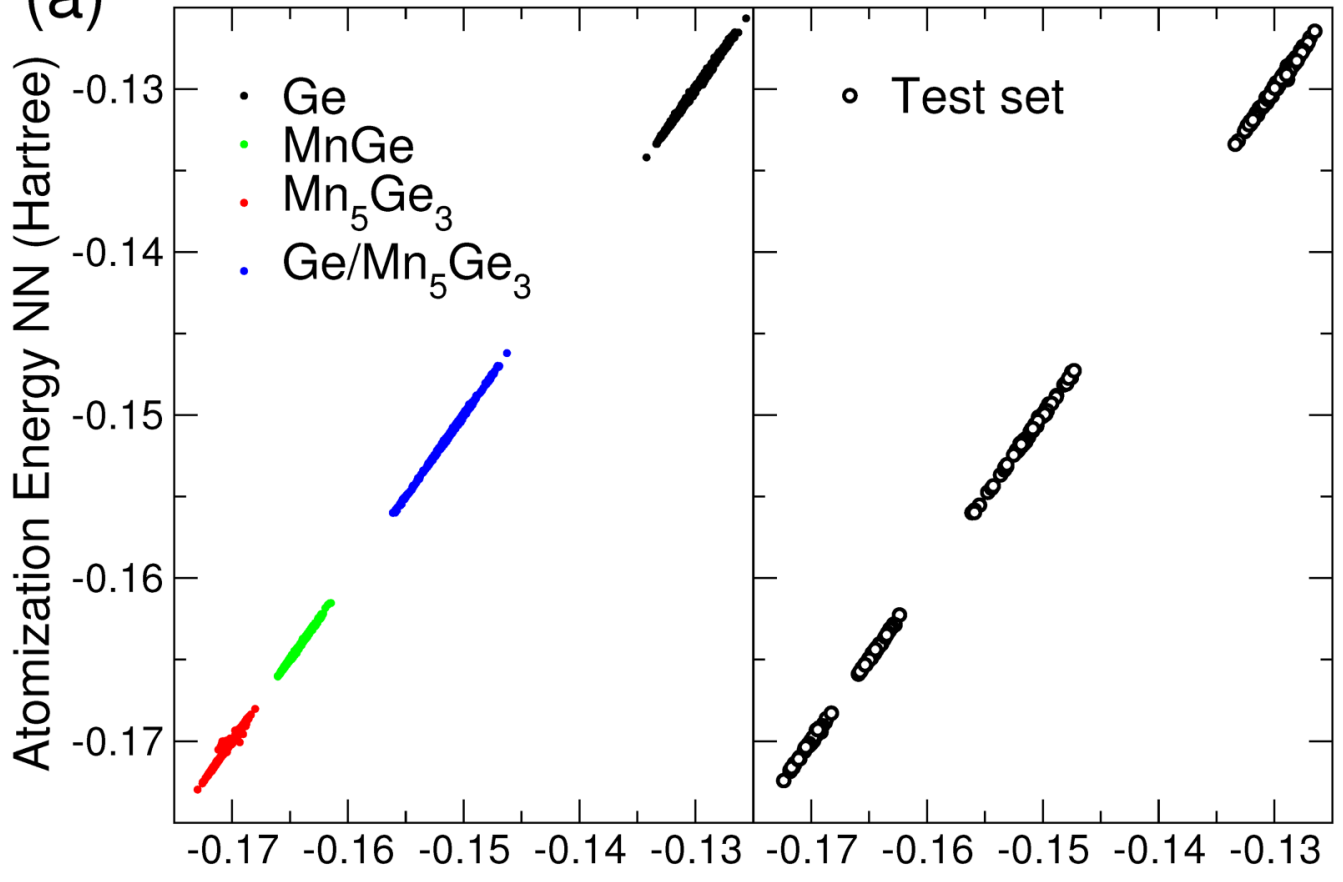

Atomization Energy (Hartree)



FIG. 3. Energies (a) and forces (b) computed by the neural network potential against the DFT reference. In panel (a), energies are shown for the training (left) and test sets (right). In panel (b), forces are shown for the test set only and are given for each separate system. Both energies and forces are in atomic units, Hartree, and Hartree/ Bohr, respectively. 
used to calculate the heat current as

$$
\mathbf{J}_{p o t}=\sum_{i} \sigma_{i} \cdot \mathbf{v}_{i}
$$

The details on how to consistently derive the two-body force and the heat current expression for multi-body potentials are provided in Ref. 61.

The MD simulations were conducted with a modified version of DLPOLY v2.1962 interfaced with RuNNer, which comprises the calculation of the heat current from the decomposition of the many-body NNP in local energy density. ${ }^{18,61}$ The same approach and software was employed to characterize thermal transport in phase change material GeTe. ${ }^{18,19}$ We considered domain sizes up to $6 \times 6 \times 6$ cubic conventional cells for $\mathrm{Ge}$ and $\mathrm{MnGe}$ and $5 \times 5 \times 6$ unit cells for solid $\mathrm{Mn}_{5} \mathrm{Ge}_{3}$. Periodic boundary conditions were applied in $x, y$, and $z$ directions. The Verlet algorithm is used to integrate the equations of motion with $1 \mathrm{fs}$ time step. In all simulations, the atomic systems were first equilibrated in a NPT (constant: number of atoms, pressure, and temperature) ensemble for $1 \mathrm{~ns}$ before being switched to a NVE (constant: number of atoms, volume, and energy) ensemble for another $1 \mathrm{~ns}$. Berendsen barostat and thermostat ${ }^{6,64}$ were used to control the pressure and the temperature of the systems during equilibration runs. Then, the following $10 \mathrm{~ns}$ simulation in NVE ensemble was taken for data production. The flux fluctuations are computed each $1 \mathrm{fs}$ and the integral is sampled over 1000 values. The correlation time upper limit of our calculations was chosen to be 50 ps. Each simulation was run for 20 times with independent initial velocity distributions. It is an inherent assumption in this study that 20 independent simulations provide a representative sample for the relevant statistical analysis. Finally, we reported the average of the 20 independent MD runs as the predicted thermal conductivity and the standard error as its uncertainty.

\section{RESULTS AND DISCUSSION}

\section{A. Structural parameters and equation of state}

We first verified that the fitted NNP reproduces the structural and vibrational properties computed by DFT of the systems used to build the training set. In Table II, we compare the lattice parameters and the bulk moduli $\left(B_{0}\right.$ of $\mathrm{Ge}, \mathrm{MnGe}$, and $\mathrm{Mn}_{5} \mathrm{Ge}_{3}$ obtained by computing the equations of state by NNP, DFT, and experiments. Equilibrium lattice parameters and $B_{0}$ are obtained by fitting the equation of state (EOS) to a Murnaghan function ${ }^{65}$

TABLE II. Lattice parameters and bulk moduli of Mn-Ge bulk materials evaluated with the NN potential and with $a b$ initio DFT calculations.

\begin{tabular}{lcccccc}
\hline \hline Species & \multicolumn{3}{c}{$\mathrm{a}(\AA)$ and c/a } & \multicolumn{3}{c}{$B_{0}(\mathrm{GPa})$} \\
\hline & $\mathrm{NN}$ & $\mathrm{DFT}$ & Expt. & $\mathrm{NN}$ & $\mathrm{DFT}$ & Expt. \\
$\mathrm{Ge}$ & 5.75 & 5.76 & 5.66 & 62.3 & 60.1 & 76.8 \\
$\mathrm{MnGe}$ & 4.75 & 4.74 & 4.80 & 113.2 & 114.2 & $\ldots$ \\
$\mathrm{Mn}_{5} \mathrm{Ge}_{3}$ & 7.15 & 7.14 & 7.18 & 104.2 & 111.0 & 110 \\
& 0.697 & 0.697 & 0.703 & & & \\
\hline \hline
\end{tabular}

(see Fig. 4). DFT results are in good agreement with experiments confirming that the adopted computational framework is reasonable to model these complex materials. ${ }^{22}$ The agreement between DFT and NNP lattice parameters is excellent to $0.01 \AA$ for the lattice parameters. In addition, NNP also provides bulk moduli in good agreement with DFT, differing at most by $6 \%$ for $\mathrm{Mn}_{5} \mathrm{Ge}_{3}$, thus suggesting that the NNP reproduces well elastic deformations, which are intrinsically connected to acoustic phonons.

\section{B. Phonons}

Producing reliable phonon dispersion relations is the first essential step for an empirical potential to predict the lattice thermal conductivity of a material. We computed the phonon dispersion relations of bulk $\mathrm{Ge}, \mathrm{Mn}_{5} \mathrm{Ge}_{3}$, and $\mathrm{MnGe}$ along a high symmetry path in the first Brillouin zone. The interatomic force constants to construct the dynamical matrix were calculated using the finite differences supercell approach, as discussed in Sec. II B. This approach is justified by the short-range nature of the forces in the systems considered; however, we were careful to verify the convergence of the DFT dispersion relations as a function of the size of the supercell used. As the NNP is short-range by construction, the calculation of the phonon dispersion relations does not suffer from size convergence issues, as long as the supercell is twice as large as the interaction cutoff radius of the NNP. NNP and DFT phonon dispersion relations, shown in Figs. 4(b), 4(d), and 4(f), are in very good agreement, especially for what concerns the acoustic branches, which provide the main contribution to heat transport. Minor discrepancies occur at higher frequency for the optical branches.

Finally, we verified that the phonons computed by NNP and DFT agree also for the $\mathrm{Ge}[111] / \mathrm{Mn}_{5} \mathrm{Ge}_{3}[001]$ superlattice used in the fitting procedure. The dispersion relations for frequencies below $5.5 \mathrm{THz}$ across the superlattice planes are shown in Fig. 5. Agreement between DFT and NN calculation remains satisfactory, although the longitudinal acoustic (LA) mode is slightly softened with the NNP with respect to DFT.

\section{Transferability of the NN potential}

Neural networks are in general a powerful approach to interpolate complex datasets but they are not reliable when it comes to extrapolation. However, NNPs may turn out transferable to phases that were not included in the training set, provided that such phases share a similar local chemical environment as the ones used for training. ' In this section, we test the transferability of our $\mathrm{Mn}_{x} \mathrm{Ge}_{y}$ NNP to the $\mathrm{Mn}_{11} \mathrm{Ge}_{8}$ phase and to $\mathrm{Ge} / \mathrm{Mn}_{5} \mathrm{Ge}_{3}$ heterostructures with different superlattice spacings [Figs. 2(b) and 2(c)].

\section{1. $\mathrm{Mn}_{17} \mathrm{Ge}_{8}$}

It is not part of the training set of the generated NNP, but since it entails structural similarities to $\mathrm{Mn}_{5} \mathrm{Ge}_{3}$, it is reasonable to expect that the NNP would perform well in reproducing its structural and vibrational properties. We evaluate the Murnaghan EOS for $\mathrm{Mn}_{11} \mathrm{Ge}_{8}$ [see Fig. 6(a)] and find it in good agreement with $a b$ initio and experimental results: the deviation in volume is only approximately $2 \%$ compared to experiment ${ }^{66}$ and less than $1.5 \%$ compared to former $a b$ initio calculations. ${ }^{24}$ The error in the bulk 



FIG. 4. Murnaghan fit of the equation of state and phonon dispersion relations of $\mathrm{Ge}[(\mathrm{a})$ and $(\mathrm{b})], \mathrm{Mn}_{5} \mathrm{Ge}_{3}[(\mathrm{c})$ and (d)], and $\mathrm{MnGe}[(\mathrm{e})$ and (f)]: comparison of results from $a b$ initio (orange solid line) and the neural network potential (black dashed line).

modulus is much larger but acceptable. The bulk modulus obtained with NNP is $142 \mathrm{GPa}$ to be compared with $105 \mathrm{GPa}$ computed by DFT. Although the NNP reproduces the structure of $\mathrm{Mn}_{11} \mathrm{Ge}_{8}$ with significantly less accuracy than for the phases included in the training set, the overall agreement with DFT is fairly good.

We further use the NNP to compute the phonon dispersion relation using a $1 \times 2 \times 1$ supercell, consisting of 152 atoms. The NNP dynamical matrix is computed by finite differences: although the supercell is fairly large, the same approach can be used to compute the phonons at the DFT level. The comparison between NNP and DFT phonon dispersion relations in the $\Gamma-X$ and $\Gamma-$ $Z$ directions is shown in Fig. 6(b), zooming into the frequencies below $2.5 \mathrm{THz}$ for the sake of clarity. The transverse acoustic (TA) phonon modes evaluated with the NN potential have slightly lower frequencies and lower group velocity (i.e., the slope of the dispersion curve) than the corresponding modes from DFT, whereas the LA mode is reproduced very well by the NNP, especially at the center zone. In turn, the low-frequency optical modes exhibit a 


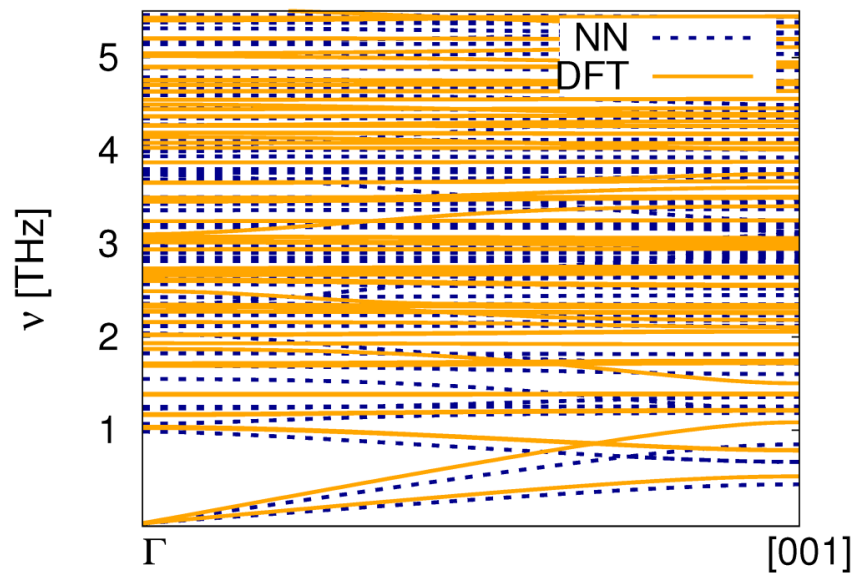

FIG. 5. Phonon dispersion relation of the interface $\mathrm{Ge}[111] / \mathrm{Mn}_{5} \mathrm{Ge}_{3}[001]$ : comparison of results achieved with ab initio DFT and the NN potential calculations.
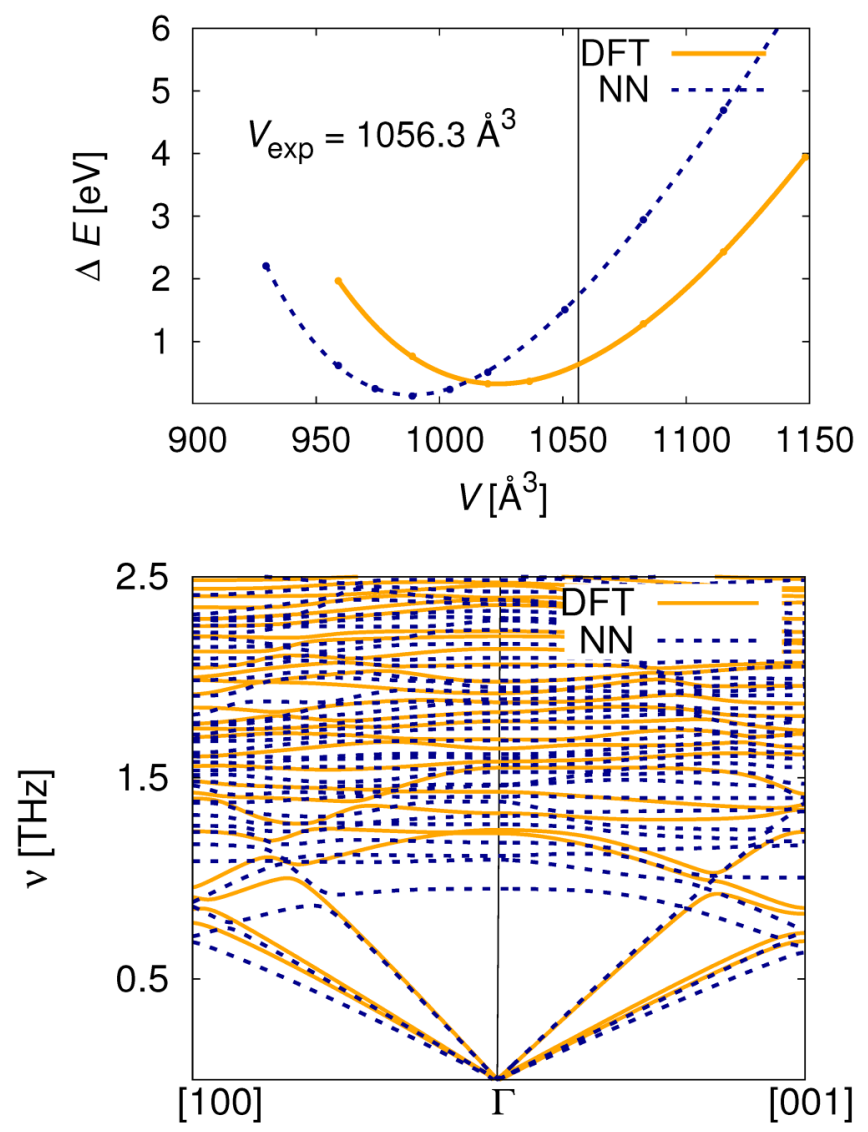

FIG. 6. Equation of state (total energy of the unit cell vs cell volume) (top panel) and phonon dispersion relation (bottom panel) of $\mathrm{Mn}_{11} \mathrm{Ge}_{8}$ : comparison of results from ab initio and the NN potential. significant shift to lower frequencies, which affects the dispersion of LA toward the edge of the Brillouin zone.

a. $\mathrm{Ge} / \mathrm{Mn}_{5} \mathrm{Ge}_{3}$ superlattices. NNP transferability has also been tested for the $\mathrm{Ge} / \mathrm{Mn}_{5} \mathrm{Ge}_{3}$ superlattices by varying the spacing between $\mathrm{Mn}_{5} \mathrm{Ge}_{3}$ layers. The interface modeled from the $\mathrm{Ge}[111]$ and the $\mathrm{Mn}_{5} \mathrm{Ge}_{3}[001]$ surface exhibits a thin Ge layer (only approximately $7 \AA$ ) and its hexagonal unit cell contains as few as 44 atoms (with only five layers of $\mathrm{Ge}$ atoms in between two $\mathrm{Mn}-\mathrm{Ge}$ phases). However, in the present research project we aim at a description of $\mathrm{Mn}-\mathrm{Ge}$ structural features like nano-columns or -clusters in a surrounding Ge matrix, i.e., much larger amount of Ge compared to $\mathrm{Mn}-\mathrm{Ge}$ phase. Therefore, we stepwise increased the Ge layer in the interfacial structure of $\mathrm{Ge}[111] / \mathrm{Mn}_{5} \mathrm{Ge}_{3}[001]$ reaching Ge layers of approximately $18 \AA$ and $28 \AA$ thickness, respectively (see Fig. 2). These heterostructures (comprising 62 and 80 atoms in the respective unit cell) were not part of the training set of the NNP. Yet, we expect NNP to provide a reliable representation of these systems, since the structure of the interfaces does not change with an increase of the Ge layer thickness.

In Fig. 7, we show the phonon dispersion relations of all three interfacial structures, computed using NNP: the top panel provides a close-up look into the low-frequency in-plane modes for the three structures, which have the same in-plane cell parameters. The slope of the TA modes depends on the thickness of the Ge layer in a nonmonotonic way, with the system with the thickest Ge layer entailing the steepest TA slopes and the highest frequencies at zone boundary. The LA mode near the Gamma point is much less affected by the Ge layer, indicating that the systems have the same longitudinal speed of sound. The cross-plane cells have different lattice parameters; thus, it makes no sense to compare the cross-plane [001] dispersion relations on the same graph: we show the low-frequency phonon dispersion relations in the three separate bottom panels in Fig. 7. For the system with the thinnest Ge layer (7 $\mathrm{A})$, the dispersion curves in the [001] direction exhibit a gap at $1 \mathrm{THz}$, which shifts at lower frequency and widens in the system with $18 \AA$ Ge layer. The gap vanishes when the Ge layer is $28 \AA$. Calculations of heterostructures with even larger lattice parameter are made accessible by the use of NNPs at a frugal computational cost. These findings indicate that NNPs can also be used to efficiently design phononic structures, such as superlattices, including the non-trivial chemical features of the interfaces within these heterostructures.

\section{Molecular dynamics and thermal conductivity}

In this section, we present the results of the thermal conductivity for the three $\mathrm{Mn}_{x} \mathrm{Ge}_{y}$ crystals used to fit the NNP, as obtained by equilibrium $\mathrm{MD}$ and the Green-Kubo approach. MD runs on supercells of $\mathrm{Ge}, \mathrm{Mn}_{5} \mathrm{Ge}_{3}$, and $\mathrm{MnGe}$ with several hundreds of atoms showed a stable behavior at $300 \mathrm{~K}$. Ge as well as $\mathrm{MnGe}$ supercell also showed stable MD runs over several ps also at elevated temperatures $(500 / 700 \mathrm{~K})$. We only observed rare instabilities for $\mathrm{Mn}_{5} \mathrm{Ge}_{3}$ at $700 \mathrm{~K}$, indicating that further high-temperature structures should be added to the NN training set, if simulations under these conditions become necessary. These simulations scale linearly with the number of atoms $(N)$ in the simulation cell, as opposed to DFT that scales like $N^{2} \log (N)$; thus, NNP can be used 
(a)

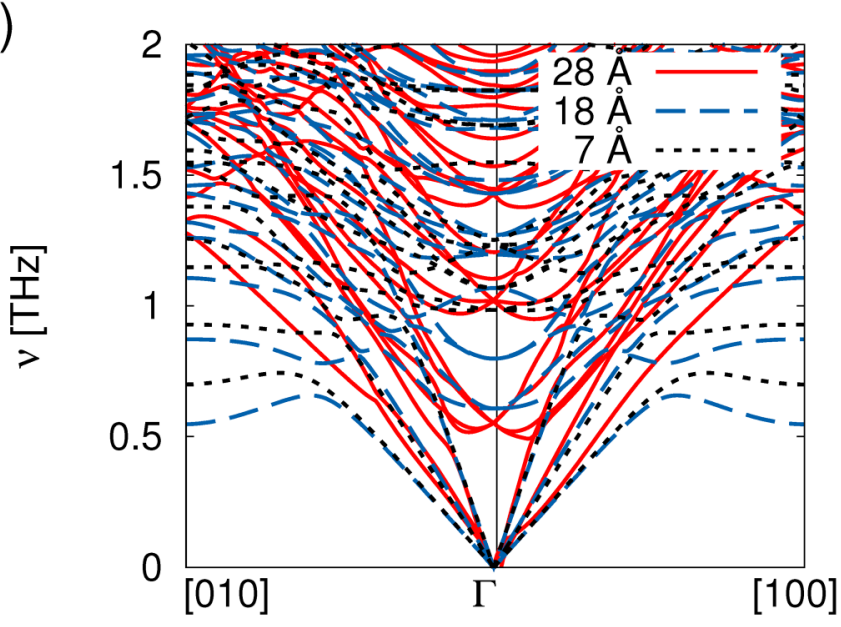

(b)

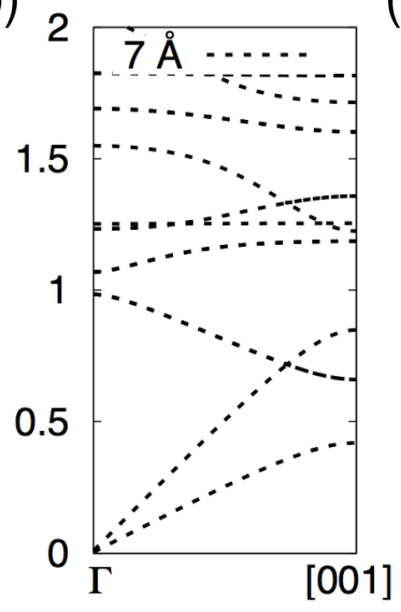

(c)

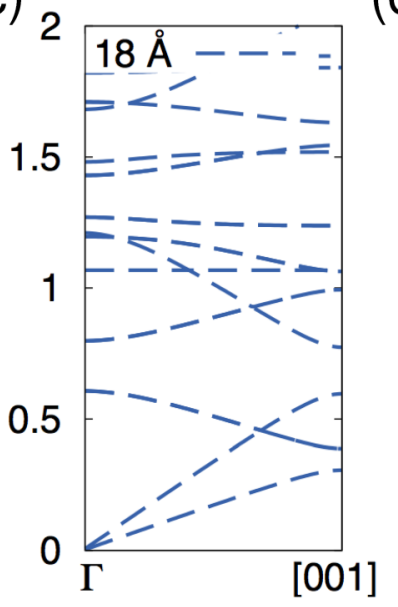

(d) 2



FIG. 7. In-plane (a) and cross-plane [(b)-(d)] phonon dispersion relation of three $\mathrm{Ge}[111] / \mathrm{Mn}_{5} \mathrm{Ge}_{3}[001]$ interfaces with of the Ge layer thickness of 7, 18, and $28 \AA$, computed using the neural network potential.

to compute the thermal conductivity by $\mathrm{MD}$, testing size convergence, and performing a sufficiently large number of runs to achieve a good statistical accuracy.

Figure 8 displays the normalized heat current autocorrelation function (HCACF) for one example run, the running integrals and their average over 20 statistically independent runs for Ge (supercell $5 \times 5 \times 5,1000$ atoms $), \mathrm{Mn}_{5} \mathrm{Ge}_{3}(5 \times 5 \times 6$ supercell, 2400 atoms $)$, and MnGe $(6 \times 6 \times 6$ supercell, 1728 atoms). All the HCACF [Figs. 8(a)-8(c)] decay rapidly to zero, thus making the evaluation of the integral in Eq. (2) relatively straightforward, with a small uncertainty on the final estimate of the thermal conductivity upon averaging. We note that the HCACFs of $\mathrm{Mn}_{5} \mathrm{Ge}_{3}$ and $\mathrm{MnGe}$ exhibit large fluctuations in the short time scale. These fluctuations, which are absent for pure Ge, are a signature of the mass difference between the elements in binary compounds, as it was formerly seen for the doped clathrate $\mathrm{Sr}_{6} \mathrm{Ge}_{46}{ }^{67}$ Figures $8(\mathrm{~d})-8(\mathrm{f})$ show the average of the integrals of the HCACF calculated for 20 independent MD simulations. For the sake of clarity, only the integrated thermal conductivity in the $z$ direction for each independent case was overlaid on the corresponding average values with the final predicted thermal conductivity and their standard error reported in each panel. For Ge and $\mathrm{MnGe}$, the calculations recover the expected isotropic value of $\kappa$ within a small error. As $\mathrm{Mn}_{5} \mathrm{Ge}_{3}$ has a hexagonal unit cell the in-plane thermal conductivity $\left(\kappa_{x y}\right)$ is different from that along the $c$ axis of the crystal $\left(\kappa_{z}\right)$. The predicted $\kappa$ of Ge, $\mathrm{Mn}_{5} \mathrm{Ge}_{3}$, and $\mathrm{MnGe}$, with supercell sizes of $5 \times 5 \times 5,5 \times 5 \times 6$ and $6 \times 6 \times 6$ u.c., are, respectively $58.9 \pm 1.30,3.3 \pm 0.15(x y) / 4.1 \pm 0.16(z)$, and $3.0 \pm 0.13 \mathrm{~W} \mathrm{~m}^{-1} \mathrm{~K}^{-1}$. The standard error for all structures in each direction is less than $5 \%$, which is small enough to be acceptable. Besides, the lattice thermal conductivity of $\mathrm{Mn}_{5} \mathrm{Ge}_{3}$ in $z$ direction $\left(4.1 \mathrm{~W} \mathrm{~m}^{-1} \mathrm{~K}^{-1}\right)$ is larger than that in the $x y$ direction (3.3 $\mathrm{W} \mathrm{m}^{-1} \mathrm{~K}^{-1}$ ), which can be related to the anisotropy of the 

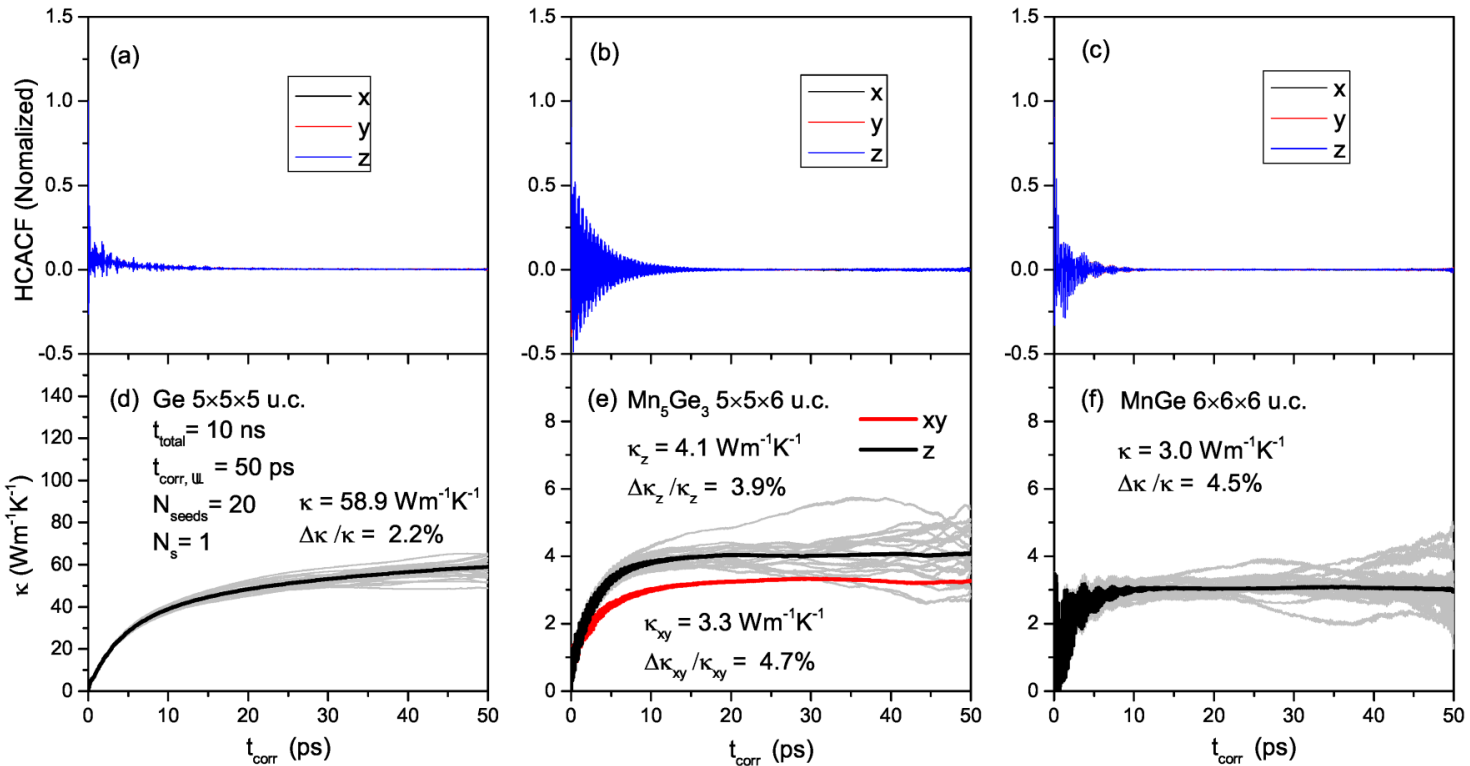

FIG. 8. Normalized heat current auto-correlation function (HCACF) of single MD runs for $\mathrm{Ge}$ (panel a), $\mathrm{Mn}_{5} \mathrm{Ge}_{3}$ (b), and $\mathrm{MnGe}$ (c). (d)-(f) Running integrals used to estimate the thermal conductivity obtained by averaging over 20 independent simulations for the same three compounds. The light-gray curves represent the thermal conductivities in the $z$ direction for each of the 20 independent simulations and the superimposed thick curves represent the corresponding average values. The obtained average thermal conductivities $\kappa$ in each direction and the corresponding standard errors $\Delta \kappa$ are also indicated in each panel along with the key details of the simulations. Since $\mathrm{Mn}_{5} \mathrm{Ge}_{3}$ is anisotropic in panel (e), the $x / y$ value of the thermal conductivity is represented in red.



FIG. 9. Variation of the EMD-predicted thermal conductivity of solid $\mathrm{Ge}$, $\mathrm{Mn}_{5} \mathrm{Ge}_{3}$, and $\mathrm{MnGe}$ at $300 \mathrm{~K}$ with the simulation domain size $\left(\mathrm{N}^{\prime}\right)$. For $\mathrm{Ge}$ and $\mathrm{MnGe}, \mathrm{N}=\mathrm{N}^{\prime}$, and for $\mathrm{Mn}_{5} \mathrm{Ge}_{3} \mathrm{~N}=\mathrm{N}^{\prime}-1$. Each bar shows the results of 20 independent simulations with standard error overlaid on the top. The red/purple dashed horizontal line shows the thermal conductivity of solid $\mathrm{Ge} / \mathrm{MnGe}$ in ferromagnetic (FM) state from DFT calculations with PBE exchange correlation functional. hexagonal structure with space group $\left[P 6_{3} / \mathrm{mcm}\right]$. However, the thermal conductivity anisotropy remains moderate, as it could be expected while considering phonon dispersions along $x y$ and $z$ directions, which do not exhibit significant differences in terms of acoustic group velocities and frequency range [see Fig. 4(b)].

As thermal conductivity calculations by MD are particularly sensitive to size effects, ${ }^{68,69}$ we have performed size convergence tests for each system considered. The results obtained using supercells with increasing size are summarized in Fig. 9. For the isotropic $\mathrm{Ge}$ and $\mathrm{MnGe}$, the data are averaged in the three directions and $\kappa$ is the result of 20 statistically independent runs with the corresponding standard error $\Delta \kappa$. For the hexagonal structure of $\mathrm{Mn}_{5} \mathrm{Ge}_{3}$, we treat $\kappa_{x y}$ and $\kappa_{z}$ independently and report both values. The values of the thermal conductivity of $\mathrm{MnGe}$ and $\mathrm{Mn}_{5} \mathrm{Ge}_{3}$ do not exhibit significant variations with size, indicating that size convergence is achieved for simulation cells of the order of 1000 atoms. For crystalline Ge, we observe light variations of thermal conductivity as a function of the system size; nevertheless, such variations remain do not exceed $5 \%$ of the value obtained with the largest supercell considered $(6 \times 6 \times 6)$, and the difference between the calculation with a $5 \times 5 \times 5$ and with a $6 \times 6 \times 6$ is well within the statistical error bars. The converged value of $\kappa$ is $54 \mathrm{~W} \mathrm{~m}^{-1} \mathrm{~K}^{-1}$, in very good agreement with experimental estimates of approximately $60 \mathrm{~W} \mathrm{~m}^{-1} \mathrm{~K}^{-1}$ for bulk $\mathrm{Ge}$ at room temperature. ${ }^{70,71}$ Besides, it is seen that by using the fitted NN potential to describe the interaction between atoms in $\mathrm{Mn}_{\mathrm{x}} \mathrm{Ge}_{1-\mathrm{x}}$ systems, the domain size has no major impact. The lattice thermal conductivities of the solid Ge, 
TABLE III. Lattice thermal conductivity of $\mathrm{Ge}$, ferromagnetic $\mathrm{MnGe}$, and $\mathrm{Mn}_{5} \mathrm{Ge}_{3}$ from equilibrium molecular dynamics with neural network potentials, compared to that obtained by DFT-BTE ( $\mathrm{Ge}$ and $\mathrm{MnGe}$ ) and experiments $(\mathrm{Ge})$.

\begin{tabular}{lccc}
\hline \hline & $\mathrm{Ge}$ & $\mathrm{MnGe}$ & $\mathrm{Mn}_{5} \mathrm{Ge}_{3}$ \\
\hline $\mathrm{NN}+$ EMD & $54.4 \pm 1.5$ & $3.1 \pm 0.09$ & $4.1 / 3.3 \pm 0.16$ \\
DFT-BTE & 49.0 & 6.0 & $\ldots$ \\
Reference & $\sim 60^{74-76}$ & $\ldots$ & $\ldots$ \\
\hline \hline
\end{tabular}

$\mathrm{Mn}_{5} \mathrm{Ge}_{3}$, and $\mathrm{MnGe}$, with largest sizes of $6 \times 6 \times 6,5 \times 5 \times 6$ and $6 \times 6 \times 6$ u.c. reached in our calculations, are $54.4 \pm 1.5,3.3 \pm$ $0.08 / 4.0 \pm 0.13(x y / z)$, and $3.1 \pm 0.09 \mathrm{~W} \mathrm{~m}^{-1} \mathrm{~K}^{-1}$, respectively. As both $\mathrm{MnGe}$ and $\mathrm{Mn}_{5} \mathrm{Ge}_{3}$ are metallic compounds (see spin densities of states in SI), their thermal conductivity would encompass the phononic contribution, computed in this work, and an electronic contribution $\left(\kappa_{e l}\right)$. The latter can be estimated through the Wiedmann-Franz law from electrical resistivity measurements: $\rho \sim 140 \mu \Omega \mathrm{cm}$ for $\mathrm{MnGe}^{72}$ and $\rho \sim 500 \mu \Omega \mathrm{cm}$ for $\mathrm{Mn}_{5} \mathrm{Ge}_{3}{ }^{73}$ Assuming the ideal value for the Lorenz number, the corresponding $\kappa_{e l}$ are 5.2 for $\mathrm{MnGe}$ and $1.5 \mathrm{Wm}^{-1} \mathrm{~K}^{-1}$ for $\mathrm{Mn}_{5} \mathrm{Ge}_{3}$, which are of the same order as the phononic contribution.

In order to validate the calculations carried out with the $\mathrm{NNP}$, the lattice thermal conductivity of Ge and MnGe computed with the EMD method is compared to that from DFT calculations. These results are summarized in Table III. For germanium, we obtain $\kappa_{N N+E M D}=54.4 \pm 1.5 \mathrm{~W} \mathrm{~m}^{-1} \mathrm{~K}^{-1}$ and $\kappa_{P B E+D F T}=49.0 \mathrm{~W} \mathrm{~m}^{-1} \mathrm{~K}^{-1}$. Both values are close to those reported in previous works (approximately $60 \mathrm{~W} \mathrm{~m}^{-1} \mathrm{~K}^{-1}$ ), ${ }^{74-76}$ which nevertheless use the local density approximation (LDA) for the exchange correlation functional. The lattice thermal conductivity of ferromagnetic MnGe from DFT-BTE calculations is $6.0 \mathrm{~W} \mathrm{~m}^{-1} \mathrm{~K}^{-1}$, which is almost twice as much as the value of $3.1 \pm 0.09 \mathrm{~W} \mathrm{~m}^{-1} \mathrm{~K}^{-1}$ from $\mathrm{NN}$ potential and EMD method. This discrepancy may probably arise from the fact that MD simulations include all order of anharmonicity, while our DFT-BTE calculations truncate the expansion of the potential to the third order, thus including only three-phonon scattering processes. In fact, recent works pointed out the importance of four-phonon scattering, especially in strongly anharmonic systems, ${ }^{77}$ which leads to substantial discrepancies between MD and BTE calculations. ${ }^{78}$ We stress that such large discrepancies do not necessarily stem from the complexity of the system or from the use of DFT. In fact, even with simple Lennard-Jones potentials BTE and MD results start to substantially diverge at relatively low temperature, where one would naively expect anharmonic lattice dynamics to be still a good approximation. ${ }^{79}$ Given the capability of NNP to accurately reproduce the phonon dispersion relations and the equation of state of $\mathrm{MnGe}$, and the inclusion of all orders of phonon-phonon scattering in MD, we tend to consider the lower value of $\kappa$ obtained by EMD the best prediction for the yet unmeasured thermal conductivity of MnGe.

\section{CONCLUSIONS}

In summary, we have shown that a NNP, trained over a relatively small set of crystalline configurations, provides a satisfactory description of the structural and vibrational properties of $\mathrm{Mn}_{x} \mathrm{Ge}_{y}$ compounds over a broad range of chemical compositions. The NNP is also able to predict reasonably well the equation of state and the phonon dispersion relations of a crystalline phase, $\mathrm{Mn}_{11} \mathrm{Ge}_{8}$, which was not used for training, and it enables the calculation of the phonon dispersion relations of $\mathrm{Ge} / \mathrm{Mn}_{5} \mathrm{Ge}_{3}$ heterostructures. In spite of numerical discrepancies between the thermal conductivity, the $\mathrm{Mn}_{x} \mathrm{Ge}_{y}$ compounds computed by DFT-based anharmonic and with the NNP, our work provides the proof of principles that the NNP can be used to reliably compute the thermal conductivity of complex systems by MD across a variety of compositions and chemical environments. This is especially important because linear-scaling MD simulations allow one to take into account phonon scattering at all orders, which is crucial to achieve accurate predictions of $\kappa$ for complex systems with strong anharmonicity. This study may be considered as a proof of principles of the transferability of NNPs to compute the thermal conductivity of complex materials over different compositions. This approach may be improved in several ways, for example, by exploring NNPs with different structures or using different symmetry functions. ${ }^{6,8,80}$ Further efforts may be exerted to improve the construction of the training database by exploring more efficiently the configurational space, so as to reduce redundancies and overfitting. ${ }^{81}$

\section{SUPPLEMENTARY MATERIAL}

The supplementary material includes the spin density of states for $\mathrm{MnGe}$ and $\mathrm{Mn}_{5} \mathrm{Ge}_{3}$ ferromagnetic compounds, and the parameter files of the neural network potential in the format interpreted by the RuNNer code.

\section{ACKNOWLEDGMENTS}

The authors thank Gabriele C. Sosso and Jinming Dong for fruitful discussions. Financial support was provided by the European Commission FP7 FET Energy Project MERGING (Grant No. 309150) and by the ANR project MESOPHON (No. ANR-15-CE30-0019). J.B. gratefully acknowledges a DFG Heisenberg professorship (Be3264/11-2, Project No. 329898176). The authors acknowledge the provision of computing facilities and support by the Rechenzentrum Garching of the Max Planck Society (MPG), the supercomputer SuperMUC at the Leibniz Rechenzentrum (Project No. pr87bi), the GENCI-IDRIS High Performance Computing resources (Grant No. A0030907186), and the "Lorraine Université computation center EXPLOR."

\section{DATA AVAILABILITY}

The data that support the findings of this study are available within the article and its supplementary material.

\section{REFERENCES}

${ }^{\mathbf{1} J . ~ B e h l e r, ~ " P e r s p e c t i v e: ~ M a c h i n e ~ l e a r n i n g ~ p o t e n t i a l s ~ f o r ~ a t o m i s t i c ~ s i m u l a t i o n s, " ~}$ J. Chem. Phys. 145, 170901-170910 (2016).

${ }^{2}$ J. Behler and M. Parrinello, "Generalized neural-network representation of high-dimensional potential-energy surfaces,” Phys. Rev. Lett. 98, 146401 (2007). 
${ }^{3}$ L. Zhang, J. Han, H. Wang, R. Car, and W. E, "Deep potential molecular dynamics: A scalable model with the accuracy of quantum mechanics," Phys. Rev. Lett. 120, 143001 (2018).

${ }^{4} \mathrm{~L}$. Bonati and M. Parrinello, "Silicon liquid structure and crystal nucleation from ab initio deep metadynamics," Phys. Rev. Lett. 121, 265701 (2018).

${ }^{5}$ A. P. Bartók, M. C. Payne, R. Kondor, and G. Csányi, "Gaussian approximation potentials: The accuracy of quantum mechanics, without the electrons," Phys. Rev. Lett. 104, 136403 (2010).

${ }^{6}$ A. P. Bartók, R. Kondor, and G. Csányi, "On representing chemical environments,” Phys. Rev. B 87, 184115-184116 (2013).

${ }^{7}$ A. V. Shapeev, "Moment tensor potentials: A class of systematically improvable interatomic potentials," Multiscale Model. Simul. 14, 1153-1173 (2016).

${ }^{8}$ A. P. Thompson, L. P. Swiler, C. R. Trott, S. M. Foiles, and G. J. Tucker, "Spectral neighbor analysis method for automated generation of quantum-accurate interatomic potentials," J. Comput. Phys. 285, 316-330 (2015).

${ }^{9}$ J. Behler, R. Martonak, D. Donadio, and M. Parrinello, "Metadynamics simulations of the high-pressure phases of silicon employing a high-dimensional neural network potential," Phys. Rev. Lett. 100, 185501 (2008).

${ }^{10}$ R. Z. Khaliullin, H. Eshet, T. D. Kühne, J. Behler, and M. Parrinello, "Graphite-diamond phase coexistence study employing a neural-network mapping of the ab initio potential energy surface," Phys. Rev. B 81, 100103 (2010).

${ }^{11}$ T. Morawietz, A. Singraber, C. Dellago, and J. Behler, "How van der Waals interactions determine the unique properties of water," Proc. Natl. Acad. Sci. U.S.A. 113, 8368-8373 (2016).

${ }^{12}$ M. A. Caro, V. L. Deringer, J. Koskinen, T. Laurila, and G. Csányi, "Growth mechanism and origin of high sp3 content in tetrahedral amorphous carbon," Phys. Rev. Lett. 120, 166101 (2018).

${ }^{13}$ V. L. Deringer, N. Bernstein, A. P. Bartók, M. J. Cliffe, R. N. Kerber, L. E. Marbella, C. P. Grey, S. R. Elliott, and G. Csányi, "Realistic atomistic structure of amorphous silicon from machine-learning-driven molecular dynamics," J. Phys. Chem. Lett. 9, 2879-2885 (2018).

${ }^{14} \mathrm{~N}$. Artrith, B. Hiller, and J. Behler, "Neural network potentials for metals and oxides-First applications to copper clusters at zinc oxide," Phys. Status Solidi B 250, 1191-1203 (2012).

${ }^{15}$ S. Gabardi, E. Baldi, E. Bosoni, D. Campi, S. Caravati, G. C. Sosso, J. Behler, and M. Bernasconi, "Atomistic simulations of the crystallization and aging of GeTe nanowires,” J. Phys. Chem. C 121, 23827-23838 (2017).

${ }^{16} \mathrm{~V}$. Quaranta, M. Hellström, and J. Behler, "Proton-transfer mechanisms at the water- $\mathrm{ZnO}$ interface: The role of presolvation," J. Phys. Chem. Lett. 8, 1476-1483 (2017).

${ }^{17} \mathrm{M}$. Gastegger, J. Behler, and P. Marquetand, "Machine learning molecular dynamics for the simulation of infrared spectra," Chem. Sci. 8, 6924-6935 (2017).

${ }^{18}$ G. C. Sosso, D. Donadio, S. Caravati, J. Behler, and M. Bernasconi, "Thermal transport in phase-change materials from atomistic simulations," Phys. Rev. B 86, 104301 (2012).

${ }^{19}$ D. Campi, D. Donadio, G. C. Sosso, J. Behler, and M. Bernasconi, "Electronphonon interaction and thermal boundary resistance at the crystal-amorphous interface of the phase change compound GeTe," J. Appl. Phys. 117, 015304 (2015).

${ }^{\mathbf{2 0}} \mathrm{R}$. Li, E. Lee, and T. Luo, "A unified deep neural network potential capable of predicting thermal conductivity of silicon in different phases," Mater. Today Phys. 12, 100181 (2020).

${ }^{21}$ M. Jamet, A. Barski, T. Devillers, V. Poydenot, R. Dujardin, P. Bayle-Guillemaud, J. Rothman, E. Bellet-Amalric, A. Marty, J. Cibert, R. Mattana, and S. Tatarenko, "High-Curie-temperature ferromagnetism in selforganized $\mathrm{Ge}_{1-x} \mathrm{Mn}_{x}$ nanocolumns," Nat. Mater 5, 653-659 (2006).

${ }^{22}$ E. Arras, I. Slipukhina, M. Torrent, D. Caliste, T. Deutsch, and P. Pochet, "First principles prediction of the metastability of the GeMn phase and its synthesis pathways," Appl. Phys. Lett. 96, 231904 (2010).

${ }^{23}$ S. Tardif, V. Favre-Nicolin, F. Lançon, E. Arras, M. Jamet, A. Barski, C. Porret, P. Bayle-Guillemaud, P. Pochet, T. Devillers, and M. Rovezzi, "Strain and correlation of self-organized $\mathrm{Ge}_{1-x} \mathrm{Mn}_{x}$ nanocolumns embedded in Ge (001)," Phys. Rev. B 82, 104101-104108 (2010).
${ }^{24}$ E. Arras, D. Caliste, T. Deutsch, F. Lancon, and P. Pochet, "Phase diagram, structure, and magnetic properties of the Ge-Mn system: A first-principles study," Phys. Rev. B 83, 174103 (2011).

${ }^{25}$ A. Spiesser, I. Slipukhina, M. T. Dau, E. Arras, V. Le Thanh, L. Michez, P. Pochet, H. Saito, S. Yuasa, M. Jamet, and J. Derrien, "Control of magnetic properties of epitaxial $\mathrm{Mn}_{5} \mathrm{Ge}_{3} \mathrm{C}_{x}$ films induced by carbon doping," Phys. Rev. B 84, 165203 (2011).

${ }^{26}$ Y. Fujishiro, N. Kanazawa, T. Shimojima, A. Nakamura, K. Ishizaka, T. Koretsune, R. Arita, A. Miyake, H. Mitamura, K. Akiba, M. Tokunaga, J. Shiogai, S. Kimura, S. Awaji, A. Tsukazaki, A. Kikkawa, Y. Taguchi, and Y. Tokura, "Large magneto-thermopower in MnGe with topological spin texture," Nat. Commun. 9, 408 (2018).

${ }^{27}$ A. Togo, L. Chaput, and I. Tanaka, "Distributions of phonon lifetimes in Brillouin zones," Phys. Rev. B 91, 094306 (2015).

${ }^{28}$ R. Kubo, "The fluctuation-dissipation theorem," Rep. Prog. Phys. 29, 255-284 (1966).

${ }^{29} \mathrm{R}$. Zwanzig, "Time-correlation functions and transport coefficients in statistical mechanics," Annu. Rev. Phys. Chem. 16, 67-102 (1965).

${ }^{30} \hat{E}$. Arras, F. Lancon, I. Slipukhina, E. Prestat, M. Rovezzi, S. Tardif, A. Titov, P. Bayle-Guillemaud, F. d'Acapito, A. Barski, V. Favre-Nicolin, M. Jamet, J. Cibert, and P. Pochet, "Interface-driven phase separation in multifunctional materials: The case of the ferromagnetic semiconductor GeMn," Phys. Rev. B 85, 115204-115210 (2012).

${ }^{31}$ J. B. Forsyth and P. J. Brown, "The spatial distribution of magnetisation density in $\mathrm{Mn}_{5} \mathrm{Ge}_{3}$," J. Phys. Condens. Matter 2, 2713-2720 (1999).

${ }^{32}$ O. L. Makarova, A. V. Tsvyashchenko, G. Andre, F. Porcher, L. N. Fomicheva, N. Rey, and I. Mirebeau, "Neutron diffraction study of the chiral magnet MnGe," Phys. Rev. B 85, 205205-205205 (2012).

${ }^{33} \mathrm{~N}$. Yamada, "Atomic magnetic moment and exchange interaction between Mn atoms in intermetallic compounds in Mn-Ge system," J. Phys. Soc. Jpn. 59, 273-288 (1990).

${ }^{34}$ J. P. Perdew, K. Burke, and M. Ernzerhof, "Generalized gradient approximation made simple," Phys. Rev. Lett. 77, 3865-3868 (1996).

${ }^{35}$ P. Giannozzi, S. Baroni, N. Bonini, M. Calandra, R. Car, C. Cavazzoni, D. Ceresoli, G. L. Chiarotti, M. Cococcioni, I. Dabo, A. Dal Corso, S. de Gironcoli, S. Fabris, G. Fratesi, R. Gebauer, U. Gerstmann, C. Gougoussis, A. Kokalj, M. Lazzeri, L. Martin-Samos, N. Marzari, F. Mauri, R. Mazzarello, S. Paolini, A. Pasquarello, L. Paulatto, C. Sbraccia, S. Scandolo, G. Sclauzero, A. P. Seitsonen, A. Smogunov, P. Umari, and R. M. Wentzcovitch, "QUANTUM ESPRESSO: A modular and open-source software project for quantum simulations of materials," J. Phys. Cond. Mat. 21, 395502 (2009).

${ }^{36} \mathrm{H}$. J. Monkhorst and J. D. Pack, "Special points for Brillouin-zone integrations," Phys. Rev. B 13, 5188-5192 (1976).

${ }^{37}$ N. Marzari, D. Vanderbilt, A. De Vita, and M. C. Payne, "Thermal contraction and disordering of the $\mathrm{Al}(110)$ surface," Phys. Rev. Lett. 82, 3296-3299 (1999).

${ }^{38}$ P. E. Blöchl, "Projector augmented-wave method," Phys. Rev. B 50, 17953-17979 (1994)

${ }^{39} \mathrm{~N}$. Holzwarth, A. R. Tackett, and G. E. Matthews, "A projector augmented wave (PAW) code for electronic structure calculations, part I: Atompaw for generating atom-centered functions," Comput. Phys. Commun. 135, 329-347 (2001).

${ }^{40}$ S. Baroni, S. de Gironcoli, A. Dal Corso, and P. Giannozzi, "Phonons and related crystal properties from density-functional perturbation theory," Rev. Mod. Phys. 73, 515-562 (2001)

${ }^{41}$ G. Kresse and J. Hafner, "Ab initio molecular dynamics for liquid metals," Phys. Rev. B 47, 558-561 (1993).

${ }^{42} \mathrm{G}$. Kresse and J. Hafner, "Ab initio molecular-dynamics simulation of the liquid-metal-amorphous-semiconductor transition in germanium," Phys. Rev. B 49, 14251-14269 (1994).

${ }^{43} \mathrm{G}$. Kresse and J. Furthmüller, "Efficiency of $a b$ initio total energy calculations for metals and semiconductors using a plane-wave basis set," Comput. Mater. Sci. 6, 15-50 (1996).

${ }^{44} \mathrm{G}$. Kresse and J. Furthmüller, "Efficient iterative schemes for ab initio total-energy calculations using a plane-wave basis set," Phys. Rev. B 54, 11169-11186 (1996). 
${ }^{45}$ P. E. Blöchl, "Projector augmented-wave method," Phys. Rev. B 50, 17953-17979 (1994)

${ }^{46} \mathrm{G}$. Kresse and D. Joubert, "From ultrasoft pseudopotentials to the projector augmented-wave method," Phys. Rev. B 59, 1758-1775 (1999).

${ }^{47} \mathrm{~J}$. Behler, "Representing potential energy surfaces by high-dimensional neural network potentials," J. Phys. Condens. Matter 26, 183001 (2014).

${ }^{48} \mathrm{~J}$. Behler, "First principles neural network potentials for reactive simulations of large molecular and condensed systems," Angew. Chem. Int. Ed. 56, 12828-12840 (2017).

${ }^{49} \mathrm{~J}$. Behler, "Atom-centered symmetry functions for constructing highdimensional neural network potentials," J. Chem. Phys. 134, 074106 (2011).

${ }^{50}$ J. Behler, "Constructing high-dimensional neural network potentials: A tutorial review," Int. J. Quantum Chem. 115, 1032-1050 (2015).

${ }^{51}$ G. C. Sosso, G. Miceli, S. Caravati, J. Behler, and M. Bernasconi, "Neural network interatomic potential for the phase change material GeTe," Phys. Rev. B 85, 174103-174113 (2012).

${ }^{52}$ G. C. Sosso, G. Miceli, S. Caravati, F. Giberti, J. Behler, and M. Bernasconi, "Fast crystallization of the phase change compound GeTe by large-scale molecular dynamics simulations," J. Phys. Chem. Lett. 4, 4241-4246 (2013).

${ }^{53}$ E. Bosoni, D. Campi, D. Donadio, G. C. Sosso, J. Behler, and M. Bernasconi, "Atomistic simulations of thermal conductivity in GeTe nanowires," J. Phys. D Appl. Phys. 53, 054001-054011 (2019).

${ }^{54}$ C. Zeng, W. Zhu, S. C. Erwin, Z. Zhang, and H. H. Weitering, "Initial stages of Mn adsorption on Ge(111)," Phys. Rev. B 70, 14-18 (2004).

${ }^{55}$ S. Olive-Mendez, A. Spiesser, L. A. Michez, V. Le Thanh, A. Glachant, J. Derrien, T. Devillers, A. Barski, and M. Jamet, "Epitaxial growth of Mn5Ge3/ Ge(111) heterostructures for spin injection," Thin Solid Films 517, 191-196 (2008).

${ }^{56}$ Y. Xie, Y. Yuan, M. Wang, C. Xu, R. Hübner, J. Grenzer, Y.-J. Zeng, M. Helm, S. Zhou, and S. Prucnal, "Epitaxial $\mathrm{Mn}_{5} \mathrm{Ge}_{3}(100)$ layer on $\mathrm{Ge}(100)$ substrates obtained by flash lamp annealing," Appl. Phys. Lett. 113, 222401-222406 (2018).

${ }^{57}$ J. B. Witkoskie and D. J. Doren, "Neural network models of potential energy surfaces: Prototypical examples," J. Chem. Theor. Comput. 1, 14-23 (2004).

${ }^{58} \mathrm{~N}$. Artrith, T. Morawietz, and J. Behler, "High-dimensional neural-network potentials for multicomponent systems: Applications to zinc oxide," Phys. Rev. B 83, 153101-153104 (2011)

${ }^{59} \mathrm{~N}$. Artrith and J. Behler, "High-dimensional neural network potentials for metal surfaces: A prototype study for copper," Phys. Rev. B 85, 045439-045513 (2012).

${ }^{60}$ T. Morawietz and J. Behler, "A density-functional theory-based neural network potential for water clusters including van der waals corrections," J. Phys. Chem. A 117, 7356-7366 (2013).

${ }^{61}$ Z. Fan, L. F. C. Pereira, H.-Q. Wang, J.-C. Zheng, D. Donadio, and A. Harju, "Force and heat current formulas for many-body potentials in molecular dynamics simulations with applications to thermal conductivity calculations," Phys. Rev. B 92, 3689 (2015).

${ }^{62}$ I. T. Todorov, W. Smith, K. Trachenko, and M. T. Dove, "Dl_poly_3: New dimensions in molecular dynamics simulations via massive parallelism," J. Mater. Chem. 16, 1911-1918 (2006).

${ }^{63}$ H. J. Berendsen, J. V. Postma, W. F. van Gunsteren, A. DiNola, and J. Haak, "Molecular dynamics with coupling to an external bath," J. Chem. Phys. 81, 3684-3690 (1984)
${ }^{64} \mathrm{~T}$. Morishita, "Fluctuation formulas in molecular-dynamics simulations with the weak coupling heat bath," J. Chem. Phys. 113, 2976-2982 (2000).

${ }^{65} \mathrm{~F}$. D. Murnaghan, "The compressibility of media under extreme pressures," Proc. Natl. Acad. Sci. U.S.A. 30, 244-247 (1944).

${ }^{66} \mathrm{~T}$. Ohba, N. Watanabe, and Y. Komura, "Temperature dependence of the lattice constants and the structure of $\mathrm{Mn}_{1} 1 \mathrm{Ge}_{8}$ at 295 and $116 \mathrm{~K}$," Acta Crystallogr. Sect. B 40, 351-354 (1984).

${ }^{67}$ J. Dong, O. F. Sankey, and C. W. Myles, "Theoretical study of the lattice thermal conductivity in Ge framework semiconductors," Phys. Rev. Lett. 86 2361-2364 (2001).

${ }^{68}$ P. K. Schelling, S. R. Phillpot, and P. Keblinski, "Comparison of atomic-level simulation methods for computing thermal conductivity," Phys. Rev. B 65, 517 (2002).

${ }^{69}$ A. J. McGaughey and M. Kaviany, "Phonon transport in molecular dynamics simulations: Formulation and thermal conductivity prediction," Adv. Heat Transfer 39, 169-255 (2006).

${ }^{70}$ C. J. Glassbrenner and G. A. Slack, "Thermal conductivity of silicon and germanium from $3 \mathrm{~K}$ to the melting point," Phys. Rev. 134, A1058-A1069 (1964).

${ }^{71}$ M. Asen-Palmer, K. Bartkowski, E. Gmelin, M. Cardona, A. Zhernov, A. Inyushkin, A. Taldenkov, V. Ozhogin, K. M. Itoh, and E. Haller, "Thermal conductivity of germanium crystals with different isotopic compositions," Phys. Rev. B 56, 9431 (1997).

${ }^{72}$ J. F. DiTusa, S. B. Zhang, K. Yamaura, Y. Xiong, J. C. Prestigiacomo, B. W. Fulfer, P. W. Adams, M. I. Brickson, D. A. Browne, C. Capan, Z. Fisk, and J. Y. Chan, "Magnetic, thermodynamic, and electrical transport properties of the noncentrosymmetric B20 germanides MnGe and CoGe," Phys. Rev. B 90, 155-214 (2014).

${ }^{73}$ R. Haug, G. Kappel, and A. Jaéglé, "Electrical resistivity studies of the system $\mathrm{Mn}_{5} \mathrm{Ge}_{3}-\mathrm{Mn}_{5} \mathrm{Si}_{3}$," Phys. Status Solidi A 55, 285-290 (1979).

${ }^{74}$ D. Broido, M. Malorny, G. Birner, N. Mingo, and D. Stewart, "Intrinsic lattice thermal conductivity of semiconductors from first principles," Appl. Phys. Lett. 91, 231922 (2007).

${ }^{75} \mathrm{~A}$. Ward and D. Broido, "Intrinsic phonon relaxation times from firstprinciples studies of the thermal conductivities of Si and Ge," Phys. Rev. B 81, 085205 (2010).

${ }^{76}$ J. Garg, N. Bonini, B. Kozinsky, and N. Marzari, "Role of disorder and anharmonicity in the thermal conductivity of silicon-germanium alloys: A firstprinciples study,” Phys. Rev. Lett. 106, 045901 (2011).

${ }^{77} \mathrm{~T}$. Feng and X. Ruan, "Quantum mechanical prediction of four-phonon scattering rates and reduced thermal conductivity of solids," Phys. Rev. B 93, 045202-045210 (2016).

${ }^{78}$ M. Puligheddu, Y. Xia, M. Chan, and G. Galli, "Computational prediction of lattice thermal conductivity: A comparison of molecular dynamics and Boltzmann transport approaches," Phys. Rev. Mater. 3, 085401 (2019).

${ }^{79}$ J. E. Turney, E. S. Landry, A. J. H. McGaughey, and C. H. Amon, "Predicting phonon properties and thermal conductivity from anharmonic lattice dynamics calculations and molecular dynamics simulations," Phys. Rev. B 79, 1537 (2009). ${ }^{80}$ Y. Zuo, C. Chen, X. Li, Z. Deng, Y. Chen, J. Behler, G. Csányi, A. V. Shapeev, A. P. Thompson, M. A. Wood, and S. P. Ong, "Performance and cost assessment of machine learning interatomic potentials," J. Phys. Chem. A 124, 731-745 (2020).

${ }^{81}$ L. Bonati, Y.-Y. Zhang, and M. Parrinello, "Neural networks-based variationally enhanced sampling,” Proc. Natl. Acad. Sci. U.S.A. 116, 17641-17647 (2019). 